\title{
Epidemiología, ideología y el discurso del desarrollo en El Salvador
}

\author{
Luis A. Avilés
}

El colonialismo no se satisface meramente con mantener al pueblo bajo su puño $y$ vaciar el cerebro de los naturales de toda forma y contenido.

Bajo un tipo de lógica perversa, se toma el pasado del pucblo, lo distorsiona, lo desfigura y lo destruye.

Frantz Fanon, Los condenados de la Tierra

La disciplina de la epidemiología está siendo testigo de un resurgimiento de debates teóricos sobre la naturaleza de la epidemiología y la función que ésta debe desempeñar en la sociedad. Dentro de esta controversia, a la cual se le ha de- nominado como "guerras de la epidemiología", un área de debate ha sido el lugar de la política, la ideología y el activismo en la investigación epidemiológica. ¿Acaso es la epidemiología (y la ciencia en general) inmune a influencias políticas e ideoló- 
gicas? ¿Pueden los epidemiólogos (y los científicos en general) ser investigadores rigurosos cuando a la vez se desempeñan como activistas sociales?

Este artículo pretende hacer una modesta contribución a este debate al investigar una de las formas en las que el ambiente institucional, bajo el cual se realizan estudios epidemiológicos (y científicos), constituye un mecanismo que condiciona cómo se concibe la epidemiología y cómo se realizan sus estudios. Más específicamente, este trabajo tiene el propósito de examinar la forma en que las instituciones de desarrollo crean un discurso que ejerce influencia sobre los estudios epidemiológicos a través de premisas ideológicas.

\section{Métodos}

El objeto de análisis de esta investigación es un estudio epidemiológico descriptivo de El Salvador, Perfil epidemiológico (Ayalde, 1994), realizado en 1994, bajo el auspicio de la Agencia Internacional para el Desarrollo (AID) del gobierno de Estados Unidos. En esta investigación, el Perfil epidemiológico es utilizado como un estudio de caso, que permite explorar las formas en que las instituciones dedicadas al desarrollo internacional ejercen influencia sobre la práctica de la epidemiología.

Este artículo recurre al análisis del discurso para identificar las formas en que la producción y circulación de un "discurso de desarrollo" ejerce influencia sobre el Perfil epidemiológico. Un discurso no es tan sólo la comunicación de pensamientos a través de palabras. Los discursos, usualmente, tienen reglas implícitas que definen a quién se le permite hablar, qué es lo que se permite y lo que no se permite que se diga, y en qué forma se dice. Estas reglas implícitas se basan en la idea de que el conocimiento $y$ el poder se implican mutuamente. El ejercicio del poder requiere de la producción de ciertos tipos de conocimiento, mientras que la producción de conocimiento requiere del ejercicio del poder para ser validado. El poder de organizaciones de desarrollo, tales como la Agencia Internacional para el Desarrollo y el Banco Mundial, requiere de la ela- boración de conocimiento sobre el desarrollo producido por profesionales que terminan justificando y legitimando las intervenciones de estas organizaciones. El análisis del discurso permite descubrir los mecanismos sutiles de la relación conocimiento-poder, que pueden pasar desapercibidos frente al investigador más riguroso. Uno de los problemas con el análisis del discurso reside en la tendencia a considerar los discursos como instrumentos de poder, independientemente de las relaciones materiales de dominación política y económica.

Las organizaciones del desarrollo han producido y circulado un discurso muy particular, el "discurso del desarrollo", el cual ha resultado ser un instrumento sumamente eficiente para ejercer poder sobre el tercer mundo (Escobar, 1995). El discurso del desarrollo circunscribe la forma en que se perciben y definen los problemas $y$, por lo tanto, limita las estrategias que se consideran $-\mathrm{y}$ las que no se consideran - para resolver los problemas del desarrollo. Este es el caso, por ejemplo, del concepto de globalización promovido por las organizaciones internacionales, las cuales definen los problemas del desarrollo bajo una perspectiva humanitaria y tecnocrática que, en consecuencia, excluye cualq̀uier alusión a asuntos más fundamentales como el poder político y económico y la desigualdad social. Analizar un informe epidemiológico como una forma de discurso implica analizar cómo se produce el informe, cuáles son sus afirmaciones científicas, qué asuntos son sistemáticamente excluidos del informe y qué conjunto de reglas dominan la producción de tales afirmaciones.

Este trabajo comienza analizando el fundamento teórico del Perfil epidemiológico, su ideología implícita y sus fallas científicas. Luego identifica los elementos de este informe que cumplen con las principales características del discurso del desarrollo, lo que define sus reglas implícitas. Al identificar el discurso de la epidemiología como el discurso del desarrollo, este trabajo intenta demostrar la influencia política e ideológica que las organizaciones de desarrollo ejercen sobre las investigaciones epidemiológicas. Finalmente, en este artículo se esbozan una serie de principios que deberían to- 
marse en consideración en la elaboración de perfiles epidemiológicos críticos y alternativos.

\section{El Perfil epidemiológico y la transición epi- demiológica}

Poco después de la firma de los acuerdos de paz de 1992, la Agencia Internacional para el Desarrollo inició un ambicioso proyecto de investigación llamado Ansal (Análisis del Sector Salud de El Salvador), que estableció las bases para una propuesta de reforma del sector salud. El objeto de estudio de este artículo, el Perfil epidemiológico, es uno de los informes de la serie Ansal. El Perfil epidemiológico de El Salvador tuvo como propósito analizar la situación de salud de la población y sus subgrupos, discutir intervenciones en salud y planificar políticas en este rubro (Ayalde, 1994). A pesar de la actual tendencia en epidemiología y salud pública a analizar las desigualdades en salud, el Perfil epidemiológico presenta una serie de estadísticas generales que ignoran completamente las desigualdades en salud y sus determinantes históricos y sociales (Cuadro 1).

La particular aversión de este informe a tratar el problema de la pobreza como un asunto de salud pública es consistente con su marco teórico, basado en la transición epidemiológica, una teoría que resulta ser algo indiferente ante las desigualdades en salud dentro de cada país. La teoría de la transición epidemiológica fue formulada por Abdel R. Omran, en 1971 (Omran, 1971), como una elaboración posterior de la teoría de transición demográfica. Los puntos básicos de esta teoría, según están presentados en el Perfil epidemiológico (Ayalde, 1994), aparentemente tomados de un libro de texto de salud internacional, aparecen a continuación.

\section{Cuadro 1}

Falta de atención a las desigualdades en salud en el Perfil epidemiológico

Cuadros incluidos en el texto

1. Tazas de crecimiento poblacional (1990-2025)

2 . Tasa de fertilidad por área de residencia (1978-1993)

$3 . \quad$ Población por sexo y regiones de salud (1992)

4. Causas principales de muerte (1992-1993)

5. Tasa de mortalidad de niños (1990): El Salvador y América Latina

6. Distribución de las principales causas de muerte por edad del niño (1993)

7. Tasa de mortalidad por edad y sexo (1975-1989)

Nota: Esta lista de cuadros no incluye los que aparecen en los apéndices del informe.

Fuente: Jaime Ayalde. Epidemiological Profile - Final Report. Ansal Scrics. San Salvador: U.S. AID, mayo, 1994.

El concepto de transición epidemiológica describe "... los patrones de salud y enfermedad dentro de las sociedades, el aumento en la expectativa de vida al nacer los cambios en el perfil epidemiológico, desde las enfermedades asociadas a las epidemias y al hambre hasta las enfermedades degenerativas y el daño a la salud causado por el hombre, tales como las llamadas causas 'externas', las cuales incluyen violencia, guerras, homicidios, suicidios y accidentes" (Ayalde, 1994, p. 10).

El factor que impulsa la transición en los países en vías de desarrollo es la ayuda internacional, otorgada por la comunidad internacional:

Los cambios ocurridos en el siglo XX, sobre todo aquellos ocurridos en los países en vías de desarrollo, han estado relacionados con los avances de la medicina y especialmente con la aplicación de nuevas intervenciones para la protección de la salud y la prevención de la enfermedad con el apoyo técnico y financiero de la comunidad internacional. Por lo tanto, puede afirmarse que el progreso ha sido alcanzado con relativa independencia de la situación social y económica de los países en vías de desarrollo (Ayalde, 1994, p. 10).

De acuerdo a esta teoría, existen tres etapas en la transición epidemiológica: primero, la etapa de las epidemias, el hambre y las guerras; segundo, la era de la disminución de las epidemias; y tercero, la era de las enfermedades degenerativas y ocasionadas por el hombre (Omran, 1971). Estos cam- 
bios ocurren impulsados por la modemización de los países (Omran, 1971).

Totalmente consistente con una teoría que afirma lo irrelevante de la situación socioeconómica, los problemas relacionados con la desigualdad de las clases sociales no se nombran, no se analizan y permanecen invisibles a través del informe. Este informe de setenta páginas no tiene un solo cuadro que incluya indicadores en salud relacionados con alguna medida de clase social, tales como educación, ocupación, ingreso o nivel de pobreza. El informe menciona el concepto de pobreza de una manera pasajera, bajo el título de "Riesgos asociados a la pobreza", en la sección de "Problemas de salud por factores de riesgo". El informe afirma que "las familias consideradas como extremadamente pobres carecen no tan sólo de ingreso, sino de servicios básicos, lo que agrava sus problemas de salud" y que "La pobreza es un factor que afecta negativamente el nivel de educación y de salud de la población" (Ayalde, 1994, p. 40- 41). Las conclusiones y recomendaciones mencionan la pobreza como una causa de desnutrición y de enfermedades infecciosas y parasitarias, pero no desempeña un papel fundamental en sus recomendaciones. Considerar la pobreza como un riesgo más resulta ser afín a una epidemiología de factores de riesgo, la cual fracasa en identificar las causas de la desigual distribución de las enfermedades, pues ignora la importancia de la estructura y la dinámica social. Al excluir deliberadamente toda consideración sobre desigualdades en salud, este informe termina apoyando el status quo, haciendo entonces de la epidemiología un instrumento al servicio de la ideología.

\section{La transición epidemiológica y la ideología del colonialismo}

Las ideologías implícitas en las teorías de la transición demográfica y epidemiológica han sido objetos de varias críticas, fundamentándose en la cuestionable validez empírica de esas teorías. Sin embargo, la incesante devoción que la teoría de la transición epidemiológica ha generado hace imperativo que se desenmascare su fundamento ideológico, lo que este artículo pretende realizar basándose en las teorías sociales de ideología. Este artículo parte de la premisa de que una adecuada reformulación del concepto de ideología todavía constituye un instrumento útil para analizar los mecanismos de dominación, a pesar de las recien- tes críticas elaboradas desde la perspectiva posmoderna. Basándose en el concepto crítico de ideología de John B. Thompson (1990), en esta investigación ideología representa "conocimiento en servicio de la dominación".

Una comparación de la teoría de transición epidemiológica y de la teoría de modernización del desarrollo internacional, revela el fundamento ideológico implícito en la teoría de Omran (Cuadro 2). Ambas teorías profesan fe en un proceso europeizado o americanizado de desarrollo en etapas, beneficioso para todos, hacia el cual todos los países convergen. La premisa ideológica principal es una forma disimulada de eurocentrismo: Europa como el modelo a emular. Ambas teorías encarnan el etnocentrismo que una vez caracterizó las formas más bárbaras de colonialismo, al cual James Blaut se refiere como a la "visión de mundo del colonizador" (Blaut, 1993).

La visión del mundo del colonizador (Cuadro 3), una manera concisa de describir la ideología del colonialismo, parte de la premisa de que el progreso requiere de la difusión de los valores y el conocimiento científico occidental hacia el resto del mundo (Blaut, 1993). La premisa análoga en la teoría de transición epidemiológica consiste en la afirmación de que los cambios epidemiológicos en las naciones en vías de desarrollo ocurren gracias al "apoyo técnico y financiero de la comunidad internacional... relativamente independiente de la situación social y económica de las naciones en vías de desarrollo" (Ayalde, 1994, p. 10). La consecuencia lógica de esta afirmación es que facilitar el apoyo técnico y financiero de la comunidad internacional —básicamente, anteriores potencias colonialistas- es, sin lugar a dudas, beneficioso para los países del tercer mundo, independientemente de su específica situación histórica, económica, social y cultural.

La teoría de la transición epidemiológica, como una ideología, funciona de la misma manera en que operan otras ideologías: legitimando y disimulando el orden social y unificando, fragmentando y reificando los procesos sociales (Thompson, 1990). Primero, la teoría de la transición epidemiológica legitima la dominación ejercida por las organizaciones de desarrollo, al presentarlas como organizaciones benévolas y con autoridad, dignas de ser apoyadas, puesto que - de acuerdo a la teoría- los países del tercer mundo sólo pueden pa- 


\section{Cuadro 2}

Teorías de transición epidemiológica y de modernización: premisas comunes

1. La transición epidemiológica (y la modernización) es un proceso en fases. Las sociedades comienzan en una etapa tradicional y terminan en una etapa moderna.

2. La transición epidemiológica (y la modernización) es un proceso homogeneizante, que produce tendencias hacia la convergencia de todas las naciones.

3. La transición epidemiológica (y la modernización) es un proceso de europeanización (o americanización).

4. La transición epidemiológica (y la modernización) es un proceso irreversible. Una vez comenzado, no puede detenerse.

5. La transición epidemiológica (y la modernización) es un proceso progresista y -a la larga - es deseable.

6. La transición epidemiológica (y la modernización) es un proceso largo y lento.

Este cuadro se basa en el ensayo de Susan Greenhalgh, "Anthropology Theorizes Reproduction: Integrating Practice, Political Economic and Feminist Perspectives", en Susan Greenhalgh (ed.), Situating Fertility: Anthropology and Demographic Inquiry, Cambridge University Press, New York, 1995, p. 6).

sar a la siguiente etapa epidemiológica a través del asesoramiento y las intervenciones de estas organizaciones.

\section{Cuadro 3}

Visión de mundo del colonizador

1. Europa progresa y se moderniza naturalmente.

2. No-Europa se mantiene estancada, tradicional y atrasada.

3. La causa básica del "progreso europeo" es la "mentalidad europea".

4. La razón para el atraso de no-Europa es la falta de ese factor intelectual o espiritual.

5. La forma normal (natural) para el progreso y modernización de no-Europa es la difusión de las ideas europeas.

6. La explotación del colonizado compensa (sólo en parte) la difusión de las ideas civilizadoras europeas.

Fuente: Blaut, J. The Colonizer's Model of the World. The Guilford Press, New York, 1993.

Segundo, la teoría de la transición epidemiológica disimula el poder de las organizaciones internacionales al ocultar, negar y nublar sus intereses políticos y económicos. Por ejemplo, durante los años de guerra en El Salvador, los proyectos de desarrollo local, auspiciados por la Agencia Internacional para el Desarrollo, tuvieron el propósi- to político explícito de socavar el apoyo comunitario a la guerrilla (Larner, 1989). En el período posterior a los acuerdos de paz, la AID ha financiado proyectos de investigación con la intención de expandir el mercado para los productos de Estados Unidos (PROFIT - Promoting Financial Investments and Transfers, 1994).

Tercero, la teoría de la transición epidemiológica unifica, a nivel de las ideas, la diversa realidad de los países del tercer mundo al proponer una sola solución a la diversidad de problemas de salud pública, la cual consiste en modernización y desarrollo. Esta unificación ideológica se hace patente en los proyectos del Banco Mundial:

De acuerdo a esta perspectiva [del Banco Mundial], el conjunto de diversas naciones, así como las miles de culturas regionales son indistinguibles $-\mathrm{y}$ los múltiples problemas sociales, económicos y políticos son meros aspectos de una misma crisis global- Esta situación —si acaso existiese- en realidad exigiría la creación de una agencia centralizada capaz de aplicar un remedio uniforme: Desarrollo (Caufield, 1996, p. 333).

Cuarto, la teoría de la transición epidemiológica puede fragmentar las oportunidades para desafiar la dominación al "expurgar el otro", según el término utilizado por Thompson (1990). 


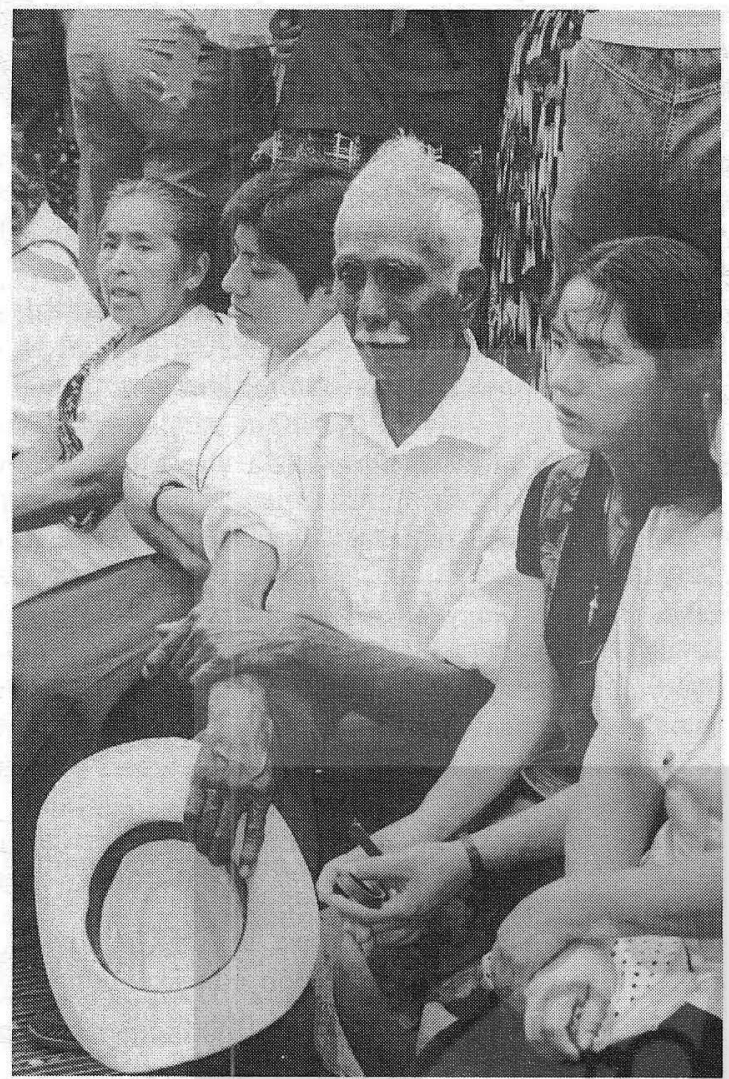

Debido a que la teoría afirma que "el progreso se ha alcanzado de una forma relativamente independiente de la situación socio-económica de los países en vías de desarrollo", la teoría de la transición epidemiológica considera como no científicos aquellos que afirman que no se puede mejorar la salud pública si no se mejora la situación social, económica y política de la población.

Quinto, la teoría de la transición epidemiológica reifica las etapas epidemiológicas, presentándolas como procesos naturales y universales que no tienen causas sociales e históricas. La ecología política de las enfermedades, una disciplina que presta particular atención a las actividades humanas en la transformación del ambiente bajo el capitalismo, ofrece una explicación para la etapa de "epidemias, hambres y guerras", que desafía la tesis de que éste es el "estado natural de la sociedad". Los relatos históricos de los diplomáticos y viajeros que visitaron El Salvador a mediados del siglo XIX, afirman que el país producía suficientes víveres como para alimentar a la población y que no existía pobreza extrema (Burns, 1984). Pero la abundancia de tierras dedicadas a la agricultura de subsistencia y la consecuente falta de mano de obra para la agricultura comercial empezó a cambiar con el ascenso al poder de los dueños de haciendas de café, durante las décadas de 1870 y 1890 (Williams, 1994). El hambre y la pobreza extrema aparecieron por primera vez en el país como consecuencia de la abolición de los ejidos (tierras comunales dedicadas a la agricultura de subsistencia), realizada por la élite con el propósito de asegurar tierras cultivables y mano de obra para aumentar la producción de café destinada al mercado internacional (Burns, 1984).

Existe un consenso entre los economistas políticos contemporáneos de Centroamérica (BulmerThomas, 1987; Weaver, 1994) en cuanto a que la polarización de las clases sociales, una de las causas fundamentales de la guerra civil de 1980-1992, no fue el resultado del estancamiento o el deterioro del crecimiento económico, sino más bien la consecuencia del exitoso crecimiento económico como resultado de la modernización de la agricultura, que ocurrió durante las décadas de 1960 y 1970. Cuando la historia demuestra que "la tendencia del crecimiento capitalista moderno acumula riqueza y poder en un polo y pobreza, coraje, y desespero en el otro" (Weaver, 1994, p. 191), se hace obvio que las epidemias, el hambre y las guerras no constituyen una etapa natural en el desarrollo epidemiológico.

En resumen, la teoría de la transición epidemiológica, aplicada a los países de la periferia de la economía mundial, no tiene otro propósito que no sea el de reforzar los patrones actuales de dominación mundial. Siguiendo la exhortación de Richard Levins de utilizar como una hipótesis inicial la premisa de que las teorías que promueven, justifican o toleran la injusticia están equivocadas, la siguiente sección explora los problemas científicos y las contradicciones con la teoría de la transición epidemiológica.

\section{Contradicciones y problemas científicos de la teoría de la transición epidemiológica}

Disciplinas académicas como la demografía histórica, la biología y la epidemiología han cuestionado la validez de la teoría de la transición epidemiológica. La teoría formulada por Omran, como una extensión de la teoría de la transición demográfica, comparte con ella sus problemas y 
contradicciones fundamentales. De acuerdo con la teoría de la transición demográfica, la transición ocurre desde "altos niveles de mortalidad y fecundidad" hacia "bajos niveles de mortalidad y fecundidad" con el surgimiento de la industrialización y la modernización. Según algunos demógrafos, no existe evidencia científica que apoye la teoría de la transición demográfica. El concepto de "transición demográfica" fue la forma en la que los demógrafos de la década de 1930 denominaron a su deus ex machina. Estudios sobre demografía histórica en Inglaterra, Francia y en los países escandinavos demuestran que el descenso en la mortalidad, en estos países, comenzó a ocurrir mucho antes que iniciara la industrialización. Más aún, un descenso uniforme en las enfermedades infecciosas y un aumento uniforme en las enfermedades no infecciosas no describe la experiencia epidemiológica de naciones tales como los Países Bajos.

Desde una perspectiva biológica, existen ciertos problemas en lo que Richard Levins ha llamado la "doctrina de la transición epidemiológica" (Levins, 1996). Fundamentando sus argumentos en ecología evolutiva, Levins afirma que la teoría de la transición epidemiológica extrapola erróneamente hacia el futuro la experiencia de los pasados cien a doscientos años. En consecuencia, esta teoría olvida que, a través de la historia, las enfermedades emergen y se disipan vinculadas a períodos de dramáticos cambios sociales, ambientales, migratorios y ecológicos (Levins, 1996). Además, debido a que la teoría de la transición epidemiológica solamente enfoca una sola especie biológica, la especie humana, sus defensores se desentienden del hecho de que las enfermedades infecciosas de las plantas y los ecosistemas emergen, se desvanecen y resurgen.

Aun dentro de la disciplina de la epidemiología, la teoría de la transición epidemiológica ha sido objeto de serias críticas difíciles de refutar. Para empezar, el concepto de la transición epidemiológica es algo cuestionable, ya que no puede ser operacionalizado sin ambigüedad. La teoría no especifica si la transición epidemiológica se refiere a todas las causas de mortalidad o a cada una de las causas específicas de mortalidad. A esto hay que añadir el problema de determinar qué enfermedades deben incluirse dentro de la "categoría algo moralista de 'enfermedades degenerativas y ocasionadas por el hombre"'. Además, esta teoría considera a los países como entidades homogéneas, ignorando y ocultando importantes diferencias epidemiológicas entre las clases sociales, los grupos étnicos o de raza, los géneros y las regiones geográficas dentro de un mismo país. Más aún, el resurgimiento de enfermedades transmisibles, tales como la tuberculosis y el sida, en ciudades de Estados Unidos y Europa, presenta resultados vergonzantes para quienes defienden esta teoría.

Incluso, la aplicación de la teoría de la transición epidemiológica a El Salvador resulta ser discutible. De acuerdo con la teoría, un signo de modernización es el nivel relativamente alto de causas "externas" de muerte, tales como la violencia, la guerra, los homicidios, los suicidios y los "accidentes". En El Salvador, posterior a los acuerdos 
de paz, las diferentes formas de violencia son tan asiduas, desde las actividades violentas de las gangas, hasta los escuadrones de la muerte (Amnesty International, 1996; Farah, 1996), que las causas "externas" de muerte son causa del mayor número de muertes en el país (Ayalde, 1994). Cuando la mayoría de las personas mueren de "enfermedades causadas por el hombre", ningún argumento teórico puede justificar la clasificación de El Salvador como un "país joven, en etapa de pre-transición" (que está a punto de iniciar su transición epidemiológica), tal y como afirma el Informe epidemiológico.

A pesar de sus objetables aseveraciones, la teoría de la transición epidemiológica continúa subsistiendo y sigue siendo ampliamente utilizada por quienes se dedican a la investigación y a la práctica de la salud pública. Utilizando como paradigma la teoría de la transición epidemiológica, se ha propuesto una "transición nutricional", aunque ésta resulta ser más cuidadosa en cuanto al carácter virtuoso y benévolo de la transición. En la práctica de la medicina interna, las categorías rígidas de las etapas epidemiológicas han fundamentado el argumento de que los internistas de los países desarrollados podrían ser más útiles en los países en transición, que en los países "pre-transicionales". Esta teoría se ha aplicado a la población hispana en Estados Unidos, argumentando que algunos segmentos de esta población "se ajustan al modelo de un país subdesarrollado en unà etapa intermedia de la transición epidemiológica". Esta afirmación tiende a enmascarar la situación de que segmentos de cualquier población étnica o racial en Estados Unidos, que vivan en los deteriorados centros urbanos de las grandes ciudades, están en mayor riesgo de contraer enfermedades debido a décadas de abandono político, explotación económica y despojo de recursos en sus comunidades (Whiteis, 1998). Al acogerse a la teoría de la transición epidemiológica, fácilmente se ignora que el proceso de acumulación de capital en el sector salud es responsable de que las comunidades de los deteriorados centros urbanos del primer mundo reciban una "medicina del tercer mundo" (Whiteis, 1998).

Debido a que la teoría de la transición epidemiológica no describe adecuadamente los patrones de enfermedad en los países de la periferia del sistema económico mundial, algunos investigadores se han visto forzados a introducir cambios y mejoras a la teoría. Por ejemplo, el término "transición epidemiológica" se ha sustituido por el de "transición de salud", con lo que se reconoce que la experiencia de América Latina no es similar a la de la transición de los países desarrollados. Algunos investigadores admiten que la transición no es unidireccional, que las etapas no son claramente distinguibles y que existen casos de transición dilatada y polarizada. Incluso, existen científicos que abogan en favor de una cuarta etapa epidemiológica y seguidores que diseñan métodos para detectarla. Si algo debe quedar claro es que cuando una teoría requiere muchos cambios para mejorar, esto es una señal de fracaso en lugar de éxito.

\section{La epidemiología como discurso del desarrollo}

Después de presentar las múltiples deficiencias de la teoría de la transición epidemiológica y la ideología del colonialismo que la misma encarna, se hace imperativo preguntarse, ¿por qué esta teoría es tan ampliamente utilizada por quienes se dedican a la investigación y a la práctica de la salud pública intemacional? El uso amplio de esta teoría responde al poder y a la influencia de las organizaciones internacionales de desarrollo. En esta sección se explica cómo el discurso del Perfil epidemiológico sigue la lógica del discurso del desarrollo, documentando la influencia del contexto institucional en la producción de las afirmaciones científicas.

\subsection{La despolitización del desarrollo}

El aspecto más sobresaliente del Perfil epidemiológico es su conspicuo silencio frente a las desigualdades en salud en El Salvador. Desde 1971, cuando Omran propuso su teoría de la transición epidemiológica, hasta 1994, cuando el Perfil epidemiológico fue publicado, una abundante serie de investigaciones documentaron los efectos nocivos de la modernización y el desarrollo capitalista al aumentar las desigualdades en salud, en la literatura de las regiones de habla hispana, portuguesa e inglesa de las Américas. Desde un punto de vista académico, excluir esta literatura es muy difícil de justificar.

Fundamentándose en datos sobre El Salvador, así como también en los de otros países latinoamericanos, el profesor Vicente Navarro ofrece una explicación que contradice a la teoría de la transición epidemiológica y al Perfil epidemiológico: 
contratos de las instituciones de desarrollo, se hace imperativo para su propia supervivencia no antagonizar o criticar las prácticas de las instituciones de desarrollo. Más aún, el conocimiento profesional del desarrollo usualmente implica la dependencia de consultores del extranjero, quienes tienen poco o ningún conocimiento de la realidad local. Cuando la mayoría de los consultores del extranjero, con poca experiencia sobre El Salvador, tienen la responsabilidad de escribir un informe luego de tres a cinco semanas de permanecer en el país (Solari, 1994), el limitado conocimiento de la realidad local difícilmente puede respaldar un reclamo que cuestione el discurso del desarrollo.

\section{Hacia la redacción de perfiles epidemioló- gicos críticos}

Después de presentar una visión de la epidemiología desde la perspectiva del colonizado y el discurso del desarrollo como un mecanismo que explica la producción y circulación de tal perspectiva, resulta deseable esbozar las bases para la redacción de un perfil epidemiológico alternativo. Los epidemiólogos pueden encontrar en uла investigación dialéctica ciertos elementos para analizar los factores precisamente omitidos por investigaciones convencionales. Tres de los principios de un modo de investigación dialéctico, presentado por Levins y Lewontin en su libro El biólogo dialéctico (Levins y Lewontin, 1985), resultan fundamentales para la redacción de perfiles epidemiológicos críticos: heterogeneidad, interconexión universal e historicidad.

El principio de heterogeneidad afirma que los objetos de investigación son internamente heterogéneos en todos su niveles. De particular relevancia para la epidemiología es el carácter heterogéneo de la población bajo estudio. Krieger y Zierler consideran que el carácter heterogéneo de la población es un aspecto fundamental de la investigación epidemiológica, debido a que claves etiológicas pueden obtenerse al "comparar la situación de salud de grupos sociales que se benefician o perjudican por el status quo, tales como empleadores y empleados, hombres y mujeres, mayorías étnicas y minorías étnicas, heterosexuales y homosexuales, y habitantes de regiones desarro-lladas y habitantes de regiones subdesarrolladas" (Krieger y Zierler, 1996). Un perfil epidemiológico adecuado de El Salvador debe presentar cómo las múltiples desigualdades en la sociedad, tales como la rígida división de clases sociales, de áreas geográficas y grupos étnicos son una de las causas fundamentales de la desigual distribución de la enfermedad.

El principio de interconexión universal insiste en la importancia de analizar los objetos de estudio en relación con procesos más amplios de los cuales forman parte. Más allá del paradigma multicausal de la epidemiología de factores de riesgo, es necesario explorar cómo las causas de la desigual distribución de la enfermedad tienen su origen en la organización y dinámica social a escala local, regional y global. Los perfiles epidemiológicos deben considerar cómo los patrones de salud y enfermedad están relacionados con las prácticas del poder de la elites locales, las prácticas excluyentes del Estado y los procesos globales de acumulación de capital. Al relacionar los procesos epidemiológicos con los procesos de acumulación de capital se hace obvio que las diferentes "etapas epidemiológicas" de la teoría de la transición epidemiológica son la justificación ideológica del desarrollo desigual de las regiones capitalistas. La creación de bolsillos de bonanza y lujo, rodeados de áreas más extensas de necesidad y deterioro, en ciudades, países y regiones, constituye una característica esencial del capitalismo. Tal y como se ha presentado en este artículo, el caso de El Salvador demuestra que la reducción de cultivos de subsistencia (con sus consecuencias para la nutrición y la salud) ha estado históricamente relacionada con la expansión de la producción de café para el mercado internacional.

El principio de historicidad afirma que cada problema tiene su historia desde dos perspectivas: la historia del problema por estudiar y la historia del pensamiento científico sobre tal problema. Los perfiles epidemiológicos deben presentar los patrones de enfermedad enmarcados dentro de la historia específica de la región y del país. Si después de décadas de la exitosa mecanización y modernización de la agricultura, en las décadas de 1960 y 1970, tres de cada diez niños en áreas rurales de El Salvador todavía sufren de desnutrición crónica (29.6 por ciento) (Asociación Demográfica Salvadoreña y Center for Disease Control and Prevention, 1999), la historicidad exige ver con escepticismo la modernización como una solución a los problemas de salud pública. Pero la historicidad también exige un examen crítico de los conceptos, los métodos y las teorías previamente utilizadas 
para describir los patrones de la enfermedad, tarea que es básicamente la que este artículo aspira a realizar.

\section{Un ejemplo de epidemiología alternativa: propuesta ciudadana}

El informe "Propuesta ciudadana por la salud" (Colegio Médico de El Salvador, 1999) demuestra la validez de la aseveración de que quienes se embarcan en modestas formas locales de resistencia política están en una posición favorable para desasir el tenaz agarre de las ideologías. Propuesta ciudadana, un informe elaborado por el militante Colegio Médico de El Salvador, un grupo que ha sido sistemáticamente excluido de los procesos de formulación de política de salud, evalúa la situación de salud en un marcado contraste con el Perfil epidemiológico. Al examinar este informe se observa que el mismo encarna los principios previamente presentados sobre la redacción de perfiles epidemiológicos críticos.

Un punto para establecer una comparación entre la Propuesta ciudadana y el Perfil epidemiológico es el conjunto de cuadros que resumen la información de ambos informes. Los primeros dos cuadros de Propuesta ciudadana presentan las primeras causas de muerte entre 1948 y 1998, concluyendo que la situación de salud del país no ha sido testigo de grandes cambios en cincuenta años. De esta comparación se concluye que cincuenta años de políticas de desarrollo han hecho muy poco para mejorar la salud de la población, lo cual constituye un ejemplo concienzudo del principio de historicidad. El tercer cuadro del informe presenta la distribución del ingreso en el país, e implícitamente aduce que la falta de mejoras sustantivas en la salud pública es resultado de la estructura social rígida. Los conceptos de pobreza y desigualdad de clase social aparecen en muchas partes del texto del informe (idesde su primera frase!), así como en los diagramas y fotografías. No obstante, el mismo presenta poca información empírica de cómo los indicadores de salud difieren de acuerdo con los grupos sociales, lo cual hace que el informe sea algo débil, en términos del principio de heterogeneidad.

Propuesta ciudadana no le imputa al subdesarrollo la mayor parte de los males de la sociedad salvadoreña y su sistema de salud; por lo tanto, se aparta de la fórmula tradicional de recomendar

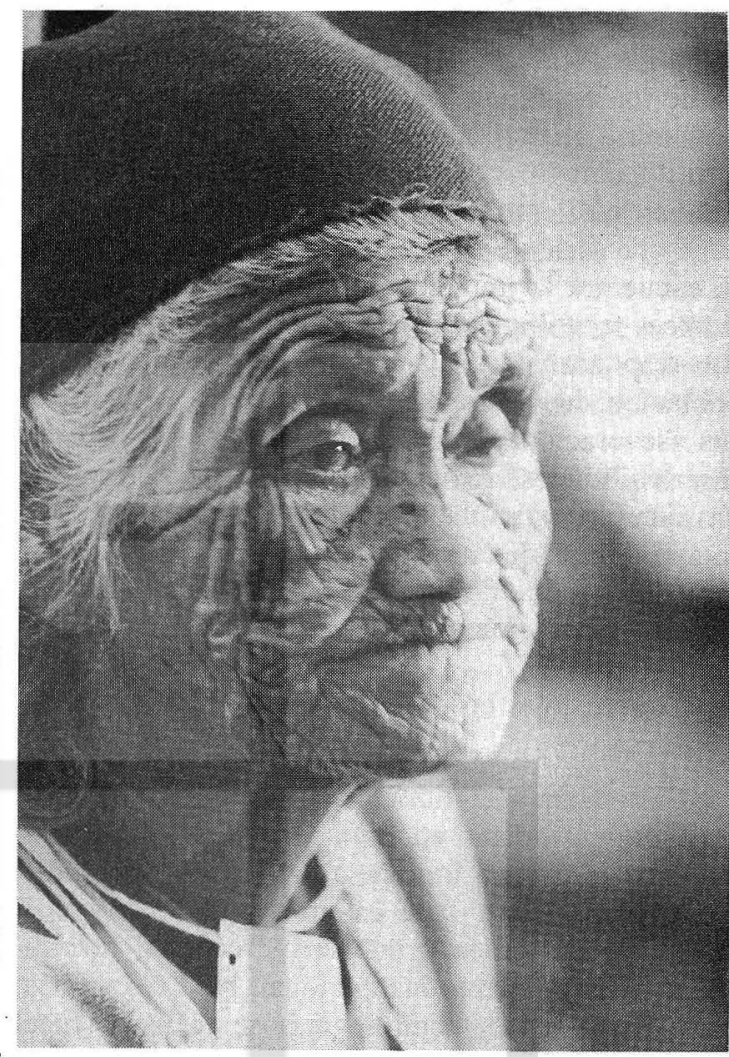

más intervenciones de instituciones de desarrollo. Este informe argumenta que las causas de las desigualdades en el sistema salud tienen sus orígenes en el modelo de desarrollo económico y las políticas de ajuste estructural, junto a la falta de voluntad política del gobierno y la preponderancia del modelo biomédico de salud. Más aún, el informe denuncia más de cuarenta años de corrupción, incompetencia administrativa y autoritarismo en el sistema de salud como una de las razones que explican la actual crisis de este sector. Propuesta ciudadana encarna el principio de conexión universal cuando relaciona la situación de salud de la población con las prácticas gubernamentales y con los modelos de desarrollo económico.

Propuesta ciudadana difiere del Perfil epidemiológico al no adecuarse a los estándares del discurso del desarrollo. Propuesta ciudadana rompe con la dependencia de expertos del extranjero y se ampara en un amplio proceso de participación, que incluye varios sectores de la sociedad. A pesar de que el Colegio Médico recibió algo de financia- 
miento de la Agencia Internacional para el Desarrollo para realizar su propuesta de reforma, aquella no tenía que rendirle cuentas a ésta por los argumentos presentados. Tal y como cualquier otro documento elaborado con base en la participación de diferentes grupos, Propuesta ciudadana tiene una serie de inconsistencias. En este informe se encuentra la impugnable afirmación de que los avances tecnológicos y la solidaridad internacional son responsables de la mejoría de la salud de la población, hecho que, en cierto modo, contradice sus aseveraciones mas fundamentales. A pesar de sus debilidades, Propuesta ciudadana constituye un ejemplo estimulante de las posibilidades para romper con el discurso del desarrollo y, por lo tanto, para producir una mejor ciencia como base para la elaboración de políticas públicas.

8. Conclusión: epidemiología, ideología y discurso

El carácter cuantitativo de la epidemiología y su enérgico énfasis en asuntos metodológicos no hace a esta ciencia inmune a las influencias políticas e ideológicas. Aun estudios epidemiológicos descriptivos contienen supuestos teóricos, formulados en el ambiente institucional, bajo el cual las investigaciones epidemiológicas se realizan. Este artículo demuestra que no es posible separar el conocimiento epidemiológico de sus implicaciones, tal como afirman algunos epidemiólogos. En el caso de las investigaciones comisionadas por las organizaciones de desarrollo, o realizadas en colaboración con ellas, el discurso de la epidemiología tiende a conformarse al discurso del desarrollo. El informe elaborado por el Colegio Médico demuestra que el activismo social no está necesariamente en conflicto con la ciencia. El Colegio Médico, al distanciarse a sí mismo del discurso del desarrollo, produjo un estudio que está más a tono con un perfil epidemiológico crítico alternativo.

Quienes abogan por una ciencia socialmente responsable creen que los epidemiólogos y otros profesionales de la salud pública tienen la responsabilidad de cuestionarse si la forma como pensamos y trabajamos refleja o contribuye a la desigualdad social. Una forma de hacer esto consiste en hacer un análisis crítico de las disciplinas de la salud pública para detectar, denunciar y eventualmente erradicar sus sesgos colonialistas y eurocéntricos, de la misma manera en que otras disciplinas académicas - como la antropología, sociolo- gía, geografía, demografía e incluso la literatura y la retórica - lo han realizado. Las actividades de los epidemiólogos socialmente responsables exigen una crítica feroz de todo lo existente, comenzando con una seria evaluación de cómo el ambiente institucional, bajo el cual se practica la epidemiología, influye sobre los conceptos, los métodos y las teorías de esta disciplina.

\section{Bibliografia}

Amnesty International. (1996). El Salvador: The Spectre of Death Squads. Amnesty International.

Asociación Demográfica Salvadoreña y Center for Disease Control and Prevention. (1999). Encuesta de Salud Familiar: FESAL-98. San Salvador.

Ayalde, J. (1994). Epidemiological Profile - Final Report. USAID. San Salvador.

Blaut, J. (1993). The Colonizer's Model of the World. The Guilford Press, New York.

Bulmer-Thomas, V. (1987). The Political Economy of Central America since 1920. Cambridge University Press, Cambridge.

Burns, B. (1984). "The Modernization of Underdevelopment: El Salvador, 1858-1931". The Journal of Developing Areas 18, pp. 293-316.

Caufield, C. (1996). Masters of Illusions: The World Bank and the Poverty of Nations. Henry Hold and Company, Inc., New York.

Colcgio Médico de El Salvador. (1999). Propuesta ciudadana por la salud. Tipografía Offsct Laser, San Salvador.

Escobar, A. (1995). Encountering Development: The Making and Unmaking of the Thirld World. Princeton University Press, Princcton.

Farah, D. (1996). "El Salvador's Government Unable to Uproots Paramilitary Groups". The Washington Post, Washington, D.C., 13 de octubre.

Ferguson, J. (1990). The Anti-Politics Machine: "Development", Depolitization, and Bureaucratic Power in Lesotho. Cambridgc Univcrsity Prcss, Cambridgc.

Kricger, N., y Zicrler, S. (1996). "What explains the public's health? - A Call for Epidemiological Theory". Epidemiology 7, pp. 107-109.

Larner, B. (1989). "AlD Strategy Targets Salvadoran Villages". The Christian Science Monitor, 29 de agosto.

Levins, R. (1996). "Why Was Public Hcalth Caught by Surprise by New and Resurgent Diseases?". En Spring Seminar - Emerging Infections Information Network. Departmento de Epidemiología y Salud Pública. Yalc University School of Medicinc. En inter- 
net: http://info.med.yale.edu/eiinet/ LevinsSeminar.html.

Levins, R., y Lewontin, R. (1985). The Dialectical Biologist. Harvard University Press, Cambridge.

Navarro, V. (1981). "The Underdevelopment of Health and the Health of Underdevelopment: An Analysis of the Distribution of Human Resources in Health in Latin America". En Vicente Navarro. Vicente Navarro, 1981. Imperialism, Health and Medicine Baywood Publishing Company, Farmingdale, New York, pp. 15-36.

Omran, A. R. (1971). "The Epidemiologic Transition: A Theory of the Epidemiology of Population Change". Milbank Memorial Fund Quarterly, pp. 509-538.

Promoting Financial Investments and Transfers (Profit). (1994). Assessment of Commercial Sector Opportunities for Family Planning and Basic Health Care in
El Salvador. Profit, Arlington, Virginia.

Solari, A. (1994). Health Sector Reform in El Salvador: Towards Equity and Efficiency. USAID, San Salvador. Thompson, J. B. (1990). Ideology and Modern Culture: Critical Social Theory in the Era of Mass Communication Polity Press, Oxford.

Weaver, F. S. (1994). Inside the Volcano: The History and Political Economy of Central America. Westview Press, Boulder.

Whiteis, D. G. (1998). "Third World Medicine in First World cities: Capital Accumulation, Uneven Development and Public Health". Social Science and Medicine 47, pp. 795-808.

Williams, R. G. (1994). States and Social Evolution: Coffee and the Rise of National Governments in Central America. The University of North Carolina Press, Chapel Hill. 